
\title{
25 Research Soure \\ First Report of Canine Bufavirus and Its Whole Genome Sequence in India
}

\section{Vishweshwar Kumar Ganji}

PV Narasimha Rao Telangana Veterinary University: Sri PV Narasimha Rao Telangana State Veterinary University

\section{Bhagyalakshmi Buddala}

PV Narasimha Rao Telangana Veterinary University: Sri PV Narasimha Rao Telangana State Veterinary University

\section{Narasimha Reddy Yella}

PV Narasimha Rao Telangana Veterinary University: Sri PV Narasimha Rao Telangana State Veterinary University

\section{Kalyani Putty ( $\square$ kalyaniputty@gmail.com )}

PV Narasimha Rao Telangana Veterinary University: Sri PV Narasimha Rao Telangana State Veterinary University https://orcid.org/0000-0002-9451-3047

\section{Research Article}

Keywords: Canine bufavirus, Protoparvovirus, Phylogenetics

Posted Date: November 17th, 2021

DOI: https://doi.org/10.21203/rs.3.rs-1059504/v1

License: (c) (i) This work is licensed under a Creative Commons Attribution 4.0 International License. Read Full License 


\section{Abstract}

Canine bufavirus (CaBuV), a novel protoparvovirus of dogs was reported only in Italy and China, till date. It was detected from dogs with enteric and respiratory symptoms and is distantly related to the human bufavirus. To explore the enteric prevalence of $\mathrm{CaBuV}$ in India, 186 diarrheic faecal samples were collected in Telangana State between 2019 and 2020. Among the samples, 4.3\% (8/186) were positive for CaBuV by PCR. Co-infection with canine parvovirus CPV-2 was seen in $75 \%(6 / 8)$ of CaBuV positive samples. The near complete genome (4292 bp) of CaBuV was amplified and reconstructed for one isolate 407/PVNRTVU/2020. Sequence alignment indicated $93.42-98.81 \%$ homology with the other available CaBuV sequences; $70.88-73.39 \%$, and $54.4-54.8 \%$ identity with human bufavirus, and CPV-2, respectively. Phylogenetic analysis showed that CaBuV 407/PVNRTVU/2020 was most closely related to CaBuV Chinese strains, together separated as Asian lineage. All Bufaviruses clustered together in one clade; however, the bat and sea otter parvoviruses also showed close relation with Bufaviruses. This first report of prevalence of $\mathrm{CaBuV}$ in India provides a good reference, emphasizes the need for further epidemiological surveillance of $\mathrm{CaBuV}$ in India and its role in canine enteritis.

\section{Introduction}

Bufaviruses (BuVs) are small, non-enveloped, single-stranded DNA viruses that belong to the family Parvoviridae, and genus Protoparvovirus [1, 2]. The genome size ranges from 4.3-4.8 kb with complex hairpin structures at the $5^{\prime}$ and $3^{\prime}$ ends. It has two open reading frames (ORFs), ORF1 and ORF2; ORF1 encodes for non-structural protein, and ORF2 encodes for capsid protein [3]. BuVs were first reported in 2012 from faecal samples of a child with acute gastroenteritis [4]. Following this, BuVs have been reported from several other humans, wild, and domestic animals [1, 5, 14, 15, 6-13]. Canine bufavirus (CaBuV) was first reported from Italy in 2016 from a litter of three mixed-breed 5-month-old puppies with canine infectious respiratory disease (CIRD) [11], followed by recently in China [16, 17]. Although the mechanism is yet unknown, CaBuVs are known to be associated with canine enteritis $[11,16,17]$.

Information on the epidemiology and genetic features of CaBuVs is rather limited; the only reports of its existence were from Italy and China [11, 16-18]. To this end, in the current study, we investigated the prevalence of $\mathrm{CaBuV}$ in India and its role in canine gastroenteritis. For this, fecal samples from dogs presented with enteritis at veterinary hospitals in Hyderabad, Telangana State, India were collected. Also, the near full-length genome of one strain was determined and characterized, providing significant reference for further studies on the evolution and epidemiology of CaBuV.

\section{Materials And Methods}

\section{Sample collection}

In total, 186 fecal samples were collected from dogs ( 1 month -10 year old) presented with clinical gastroenteritis to Veterinary hospitals in Hyderabad, Telangana State, India during 2019-2020. Of the total 
number of samples, 138 were from puppies (<1-year-old), and 48 were from adult dogs (>1-year-old) (Table S1). Informed consents were obtained from all animal owners. The fecal samples were collected in sterile centrifuge tubes using rectal swabs and stored at $-20^{\circ} \mathrm{C}$ until used.

\section{DNA extraction and PCR amplification}

The rectal swabs were homogenized in $3 \mathrm{ml}$ of $0.1 \mathrm{M} \mathrm{PBS}(\mathrm{pH} 7.4)$ containing antibiotics $(100 \mathrm{lU} / \mathrm{ml}$ Benzyl Penicillin, $100 \mu \mathrm{g} / \mathrm{ml}$ Streptomycin sulphate), centrifuged at $6000 \mathrm{rpm}$ for $10 \mathrm{~min}$ at $4^{\circ} \mathrm{C}$, and the supernatant filtered through $0.22 \mu$ syringe filter was used for further analysis. The viral DNA was extracted using phenol chloroform and isoamyl alcohol method as described by Sambrook and Russel [19]. The extracted DNA was stored at $-20^{\circ} \mathrm{C}$ until use.

For detection of CaBuV, real-time PCR was performed as described before [11]. The CaBuV positive samples were screened for coinfections with other canine enteric viruses namely, CPV-2 [20], CAdV-2 [21], CDV [22], CCoV [23], CAstV [24], CRV [25]. One of the PCR positive samples was selected for amplifying complete CaBuV genome using five different primer pairs as described before [26] with slight modifications in reaction conditions (Table S2). PCR products were purified using a DNA purification kit (Qiagen, USA) following the manufacturer's instructions, and were sequenced at sequencing facility, Bioserve, Pvt. Ltd, Hyderabad, India. The PCR products were sequenced twice. Near complete genome of the CaBuV strain 407/PVNRTVU/2020 was assembled using SeqMan software (DNASTAR). ORFs were identified using the NCBI ORF finder (https://www.ncbi.nIm.nih.gov/orffinder/).

\section{Phylogenetic and recombination analysis}

Sequence identity was analysed by multiple sequence BLAST analysis of nucleotide and amino acids. Multiple sequence alignment of the whole genome was performed by CLUSTALW algorithm of MEGAX software followed by phylogenetic tree reconstruction [27]. The phylogenetic tree was generated using the Maximum Likelihood statistical method with the tamura-3-parameter model. The credibility of the phylogenetic tree was tested by applying a bootstrap test with 1000 replicates.

The recombination analysis tool (RAT), and recombination detection program (RDP) package Beta 4.101 were used for identification of recombinant sequences in default mode. A recombination event with a significance of $p<0.01$ in at least four out of nine selected algorithms: RDP, GENECONV, Boot-Scan, Maxchi, Chimaera, SiScan, 3Seq, LARD, and PhylPro was considered to be reliable [28]. RAT is a distancebased method of recombination detection platform [29].

\section{Results}

\section{Detection of CaBuV in fecal samples}

Of the 186 samples screened by real-time PCR, eight were found positive for CaBuV which accounts for a prevalence rate of $4.3 \%$. Of these, six samples were from diarrheic puppies ( $<1$ year old) and two samples were from diarrheic adult dogs (>1 year old). Seven of the eight CaBuV positive samples had co 
infections with other enteric viruses such as canine parvovirus CPV-2, canine adeno virus-2, and canine astrovirus; and negative for canine coronavirus, and canine distempervirus (Table 1).

\section{CaBuV genome characterization}

The near complete genome sequence of CaBuV isolate 407/2020/PVNRTVU was obtained by PCR amplification (Fig. 1a) followed by sequencing and uploaded to GenBank (accession number:

MZ574435). The genome size obtained was $4292 \mathrm{nt}$ in length; the size of ORF-1 and ORF-2 was $1917 \mathrm{nt}$ and $2178 \mathrm{nt}$, respectively (Fig. 1b). The length of NS1, VP1, and VP2 was $1917 \mathrm{nt}$ (638 amino acids), $2133 \mathrm{nt}$ (710 amino acids), and $1707 \mathrm{nt}$ (568 amino acids), respectively (Fig. 1b). When compared with the other reference CaBuV strains, the similarity of complete sequences was $93.42-98.81 \%$ (Table $2 \mathrm{a}$ ). Likewise, the nucleotide similarity of NS1, VP1, and VP2 was $96.48-98.13 \%, 89.91-99.48 \%$, and $88.19-$ $99.47 \%$, respectively (Table $2 \mathrm{~b}$ ); and the amino acid similarity of NS1, VP1, and VP2 was $94.98-97.81 \%$, 94.79-99.72\%, and $94.19-99.82 \%$, respectively (Table $2 b$ ). Various novel mutations were noticed in the NS1, VP1, and VP2 coding regions with 23, 8, and 7 synonymous mutations; and 13, 2, and 1 nonsynonymous substitution sites (Table 3). Compared to the other members of Parvoviridae family including other $\mathrm{CaBuVs}$, the similarity of the complete sequence obtained in the current study was $39.5 \%$ $-98.81 \%$ (Table 2a).

\section{Phylogenetic and recombination analysis}

Phylogenetic analysis was performed with the near complete genome sequence in this study with other publicly available CaBuV sequences and other protoparvovirus sequences available in GenBank. It can be seen that the CaBuV from India was more closely related to Chinese isolates, together separated as Asian lineage (Fig. 2a). Further, it can be seen that CaBuVs show closest relationship with bat and sea otter parvoviruses in addition to all other Bufaviruses (human, porcine, rat and megabat), whereas, canine and avian parvoviruses together formed a separate branch (Fig. 2b).

To analyze whether recombination played a role in CaBuV evolution, CPVs, CaBuVs, HuBuVs, and PoBuVs, megabat BuV, rat BuV, bat BuV, avian BuV, human cutavirus, and sea otter parvovirus were analyzed by RAT and RDP software with multiple algorithms. Nine algorithms (RDP, GENECONV, BootScan, Maxchi, Chimaera, SiScan, 3Seq, LARD, and PhylPro) were used to predict potential recombination events between the input sequences. Even though no recombination events could be found in the CaBuVs, it can be noted that major recombination events possibly drove evolution of other BuVs in the Protoparvovirus genus (Fig. 3).

\section{Discussion}

$\mathrm{CaBuV}$ is a novel protoparvovirus first identified from an outbreak of CIRD [11]. Since then, only handful of studies from a few countries (Italy, Hungary, and China) reported its prevalence [11, 16-18]. We report $\mathrm{CaBuV}$ prevalence in India for the first time. Varying prevalence rates of this virus have been reported so far ranging from $1.74-42.15 \%[11,16-18,30]$. Our study reports a prevalence rate of $4.3 \%$. It has been suggested that geographical, temporal, and target canine population included in the studies can affect 
the prevalence rates [18]. A statistically non-significant correlation of CaBuV with enteritis was reported before; although non-significant the authors noticed higher loads of $\mathrm{CaBuV}$ in diarrheic samples than in controls $[11,18]$. Moreover, our study along with another study where prevalence of CaBuV was studied extensively in diarrheic dogs, stresses the role of $\mathrm{CaBuV}$ in causing enteritis [16]. We have noticed that seven out of eight CaBuV positive samples had co infections with other enteric viruses with CPV-2 being the predominant co infection (6/8) followed by CAstV (2/8), and CAdV-2 (2/8). Similar to our findings few other studies also reported CPV-2 to be the major co-infecting agent with CaBuV with a prevalence of CPV-2 ranging from $87.5 \%-100 \%[17,18]$. CPV-2 is a well-known viral agent involved in severe gastroenteritis in dogs, especially pups. Further experiments on the pathogenicity studies shall throw light on the role of co-infections in increased severity of gastroenteritis morbidity and mortality in dogs.

We have reconstructed near complete genome of one isolate of CaBuV detected in Indian dog population. Comparison and analysis with the other publicly available CaBuV sequences revealed NS1 to be the most conserved gene and VP2 to be the most variable, both at the level of nucleotide (NS1: $96.48 \%-98.13 \%$; VP2: 88.19\%-99.47\%) and amino acids (NS1: 94.98\%-97.81\%; VP2: 94.19\%-99.82\%). Based on the phylogeny reconstructed with the whole genome, the current $\mathrm{CaBuV}$ isolate clustered with $\mathrm{CaBuV}$ strains identified in China. Our analysis differentiates all the available CaBuV sequences into two groups: Asia and Europe. Being a DNA virus, parvoviruses exhibit low mutation rate [17]. The genetic grouping observed in the current study indicates unique evolutionary forces driving evolution of CaBuVs. Furthermore, CaBuV 407/2020/PVNRTVU showed highest identity (77.89\%) with megabat bufavirus strain, followed by bat parvovirus $(70.34-79.5 \%)$, porcine bufavirus $(73.16-73.4 \%)$, human bufavirus (70.92-73.39\%), sea otter parvovirus (72.7\%), rat bufavirus (63\%), canine parvovirus (54.4-54.8\%), and avian parvovirus (39.5-40\%). Pairwise comparison of each individual NS1, VP1, and VP2 protein coding regions of 407/2020/PVNRTVU revealed presence of 13,2 , and 1 unique non-synonymous substitutions when compared to other available CaBuV sequences. Understanding the biological significance of these mutations by future experimental studies can elucidate the role of these mutations (if any) in driving the evolution of this virus and adaptation to local host populations.

In summary, the first report of prevalence and complete genomic sequence of CaBuV isolate from India was obtained and genetically analyzed in our study. Indian isolate was grouped with CaBuVs isolated from China. The current study forms a foundation to further studies on the epidemiology and genetic diversity of CaBuV in India.

\section{Declarations}

\section{Acknowledgements}

We wish to express our gratitude to the animal owners who participated in the rectal swab specimen's collection.

\section{Funding}


The authors acknowledge Department of Biotechnology (No.BT/ADV/Canine Health/TANUVAS), India for the financial support.

\section{Authors' contributions}

KP conceived and designed the study. VG, BBL performed the experiments. KP, VG, YNR analyzed the data and wrote the manuscript. The author(s) read and approved the final manuscript.

\section{Availability of data and materials}

The data supporting the conclusions of this article are included within the article.

\section{Ethics approval}

This research did not involve any human participants or experiments on live animals. All the work has been performed in accordance with standard operating procedures adopted by the Department of Veterinary Biotechnology, PVNRTVU, Hyderabad, India. Before the sample collection, all the guardians of the subjects were informed the purpose of this study and consent was obtained.

\section{Conflict of interest}

The authors declare no competing interests with respect to the research, authorship, and/or publication of this article.

\section{References}

1. Hargitai R, Pankovics P, Kertész AM, et al (2016) Detection and genetic characterization of a novel parvovirus distantly related to human bufavirus in domestic pigs. Arch Virol 161:1033-1037. https://doi.org/10.1007/s00705-015-2732-4

2. Huang H, Li Y, Wang W, et al (2020) Detection and molecular characterization of novel porcine bufaviruses in Guangxi province. Infect Genet Evol 82:. https://doi.org/10.1016/j.meegid.2020.104286

3. Vaïsänen E, Paloniemi M, Kuisma I, et al (2016) Epidemiology of two human protoparvoviruses, bufavirus and tusavirus. Sci Rep 6:1-8. https://doi.org/10.1038/srep39267

4. Phan TG, Vo NP, Bonkoungou IJO, et al (2012) Acute Diarrhea in West African Children: Diverse Enteric Viruses and a Novel Parvovirus Genus. J Virol 86:11024-11030. https://doi.org/10.1128/jvi.01427-12

5. Altay A, Yahiro T, Bozdayi G, et al (2015) Bufavirus genotype 3 in Turkish children with severe diarrhoea. Clin Microbiol Infect 21:965.e1-965.e4. https://doi.org/10.1016/j.cmi.2015.06.006

6. Huang DD, Wang W, Lu Q Bin, et al (2015) Identification of Bufavirus-1 and Bufavirus-3 in Feces of Patients with Acute Diarrhea, China. Sci Rep 5:. https://doi.org/10.1038/srep13272 
7. Smits SL, Schapendonk CME, van Beek J, et al (2014) New viruses in idiopathic human diarrhea cases, the Netherlands. Emerg Infect Dis 20:1218-1222. https://doi.org/10.3201/eid2007.140190

8. Yahiro T, Wangchuk S, Tshering K, et al (2014) Novel human bufavirus genotype 3 in children with severe diarrhea, Bhutan. Emerg Infect Dis 20:1037-1039. https://doi.org/10.3201/eid2006.131430

9. Diakoudi G, Lanave G, Capozza P, et al (2019) Identification of a novel parvovirus in domestic cats. Vet Microbiol 228:246-251. https://doi.org/10.1016/j.vetmic.2018.12.006

10. Handley SA, Thackray LB, Zhao G, et al (2012) Pathogenic simian immunodeficiency virus infection is associated with expansion of the enteric virome. Cell 151:253-266. https://doi.org/10.1016/j.cell.2012.09.024

11. Martella V, Lanave G, Mihalov-Kovács E, et al (2018) Novel Parvovirus Related to Primate Bufaviruses in Dogs. Emerg Infect Dis 24:1061. https://doi.org/10.3201/EID2406.171965

12. Melegari I, Di Profio F, Palombieri A, et al (2019) Molecular detection of canine bufaviruses in wild canids. Arch Virol 164:2315-2320. https://doi.org/10.1007/s00705-019-04304-y

13. Sasaki M, Orba Y, Anindita PD, et al (2015) Distinct lineages of bufavirus in wild shrews and nonhuman primates. Emerg Infect Dis 21:1230-1233. https://doi.org/10.3201/eid2107.141969

14. Sasaki M, Gonzalez G, Wada Y, et al (2016) Divergent bufavirus harboured in megabats represents a new lineage of parvoviruses. Sci Reports 201661 6:1-8. https://doi.org/10.1038/srep24257

15. Yang S, Liu D, Wang Y, et al (2016) Bufavirus Protoparvovirus in feces of wild rats in China. Virus Genes 52:130-133. https://doi.org/10.1007/s11262-015-1262-1

16. Li J, Cui L, Deng X, et al (2019) Canine bufavirus in faeces and plasma of dogs with diarrhoea, China. Emerg. Microbes Infect. 8:245-247

17. Sun W, Zhang S, Huang H, et al (2019) First identification of a novel parvovirus distantly related to human bufavirus from diarrheal dogs in China. Virus Res 265:127-131. https://doi.org/10.1016/j.virusres.2019.03.020

18. Di Martino B, Sarchese V, Di Profio F, et al (2021) Genetic heterogeneity of canine bufaviruses. Transbound Emerg Dis 68:802-812. https://doi.org/10.1111/tbed.13746

19. Sambrook J, Russell DW (2001) Molecular cloning: a laboratory manual. Cold Spring Harbor Laboratory Press

20. Decaro N, Elia G, Martella V, et al (2005) A real-time PCR assay for rapid detection and quantitation of canine parvovirus type 2 in the feces of dogs. Vet Microbiol 105:19-28. https://doi.org/10.1016/j.vetmic.2004.09.018

21. Balboni A, Dondi F, Prosperi S, Battilani M (2015) Development of a SYBR Green real-time PCR assay with melting curve analysis for simultaneous detection and differentiation of canine adenovirus type 1 and type 2. J Virol Methods 222:34-40. https://doi.org/10.1016/j.jviromet.2015.05.009

22. Elia G, Decaro N, Martella V, et al (2006) Detection of canine distemper virus in dogs by real-time RTPCR. J Virol Methods 136:171-176 
23. Decaro N, Pratelli A, Campolo M, et al (2004) Quantitation of canine coronavirus RNA in the faeces of dogs by TaqMan RT-PCR. J Virol Methods 119:145-150.

https://doi.org/10.1016/j.jviromet.2004.03.012

24. Martella V, Moschidou P, Lorusso E, et al (2011) Detection and characterization of canine astroviruses. J Gen Virol 92:1880-1887. https://doi.org/10.1099/vir.0.029025-0

25. Logan C, O'Leary JJ, O'Sullivan N (2006) Real-time reverse transcription-PCR for detection of rotavirus and adenovirus as causative agents of acute viral gastroenteritis in children. $\mathrm{J}$ Clin Microbiol 44:3189-3195. https://doi.org/10.1128/JCM.00915-06

26. Wang Y, Guo X, Zhang D, et al (2020) Genetic and phylogenetic analysis of canine bufavirus from Anhui Province, Eastern China. Infect Genet Evol 86:104600.

https://doi.org/10.1016/J.MEEGID.2020.104600

27. Kumar S, Stecher G, Li M, et al (2018) MEGA X: Molecular evolutionary genetics analysis across computing platforms. Mol Biol Evol 35:1547-1549. https://doi.org/10.1093/molbev/msy096

28. Martin DP, Murrell B, Golden M, et al (2015) RDP4: Detection and analysis of recombination patterns in virus genomes. Virus Evol 1:. https://doi.org/10.1093/VE/VEV003

29. Etherington GJ, Dicks J, Roberts IN (2005) Recombination Analysis Tool (RAT): a program for the high-throughput detection of recombination. Bioinformatics 21:278-281. https://doi.org/10.1093/BIOINFORMATICS/BTH500

30. Shao R, Zheng F, Cai S, et al (2020) Genomic sequencing and characterization of a novel group of canine bufaviruses from Henan province, China. Arch Virol 165:2699-2702. https://doi.org/10.1007/s00705-020-04785-2

\section{Tables}

Due to technical limitations, table 1 to 3 xlsx are only available as a download in the Supplemental Files section.

\section{Figures}


A

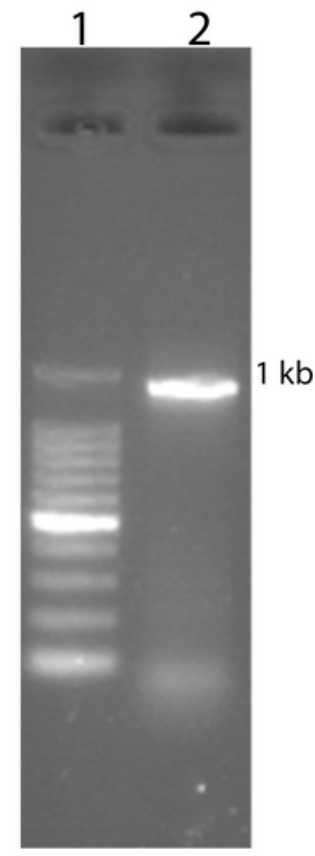

B

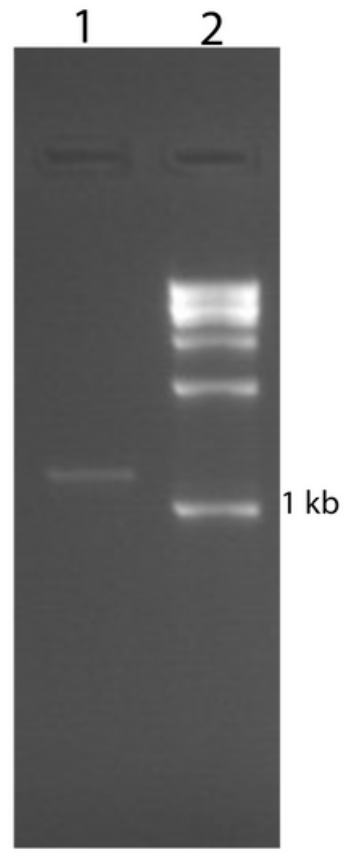

C

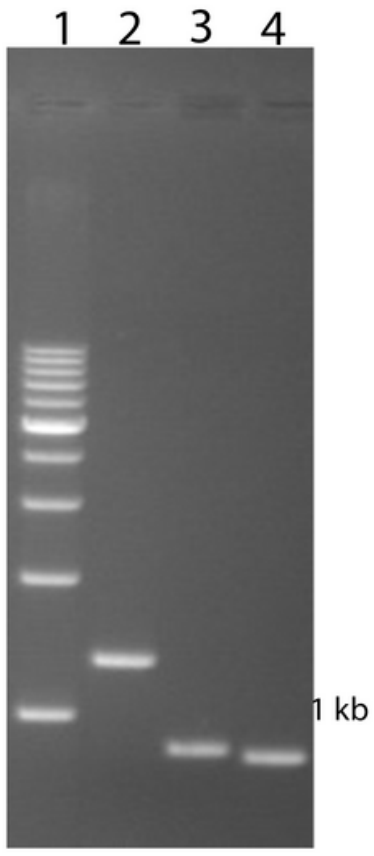

$1 a$

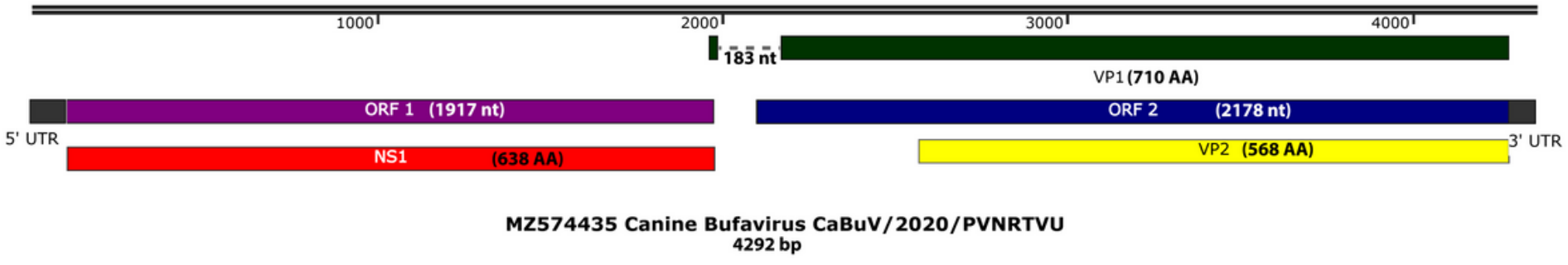

$1 b$

\section{Figure 1}

1a PCR amplification of different segments of CaBuV for sequencing. Panel A lane 1 shows 100 bp DNA marker, lane 2 shows the amplicon of size 952 bp for primer set 1. Panel B lane 1 shows the amplicon of size 1183 bp for primer set 2, lane 2 shows 1 Kb DNA marker. Panel C lane 1 shows 1 Kb DNA marker, lane 2 shows the amplicon of size 1351 bp for primer set 3, lane 3 shows the amplicon of size $848 \mathrm{bp}$ for primer set 4, lane 4 shows the amplicon of size $815 \mathrm{bp}$ for primer set $5.1 \mathrm{~b}$ Graphical representation of CaBuV (MZ574435 isolate) whole genome. 
$2 b$

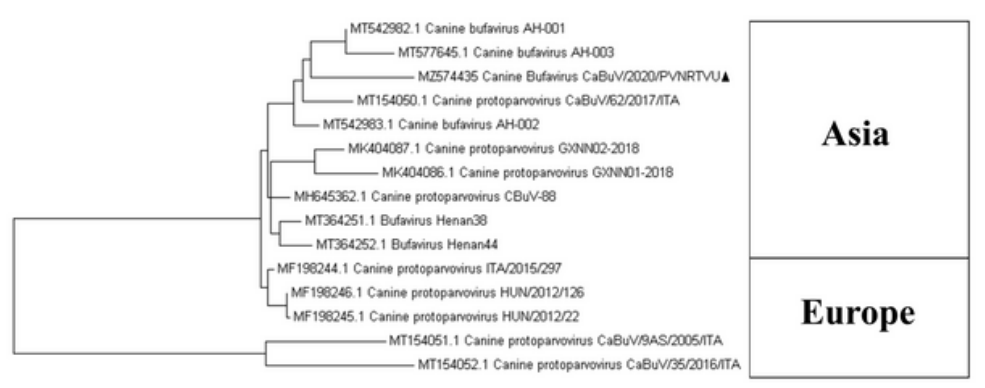

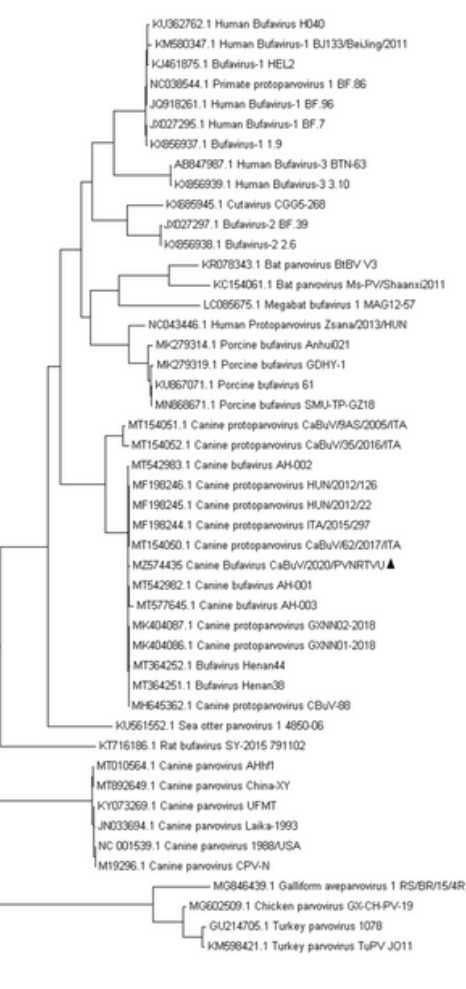

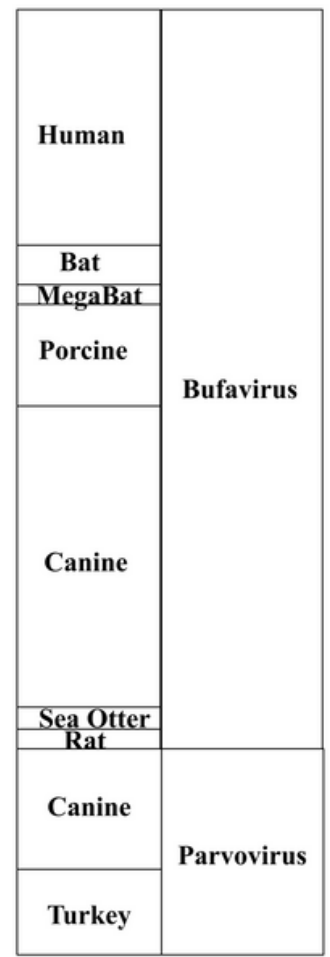

Figure 2

2a Phylogenetic tree of CaBuVs based on whole genome sequence. A total of 15 whole genome of CaBuV isolates published earlier in GenBank were used as reference to reconstruct the phylogenetic tree using maximum likelihood as statistical method, test of phylogeny as bootstrap with value of 1000 replicates, model as tamura-3-parameter. The isolate from this study is marked with a dark triangle. $2 \mathrm{~b}$ Phylogenetic tree of Parvoviridae family based on whole genome sequence. A total of 46 whole genome of Parvoviridae family isolates infecting different species published earlier in GenBank were used as reference to reconstruct the phylogenetic tree using maximum likelihood as statistical method, test of phylogeny as bootstrap with value of 1000 replicates, model as tamura-3-parameter. The isolate from this study is marked with a dark triangle. 


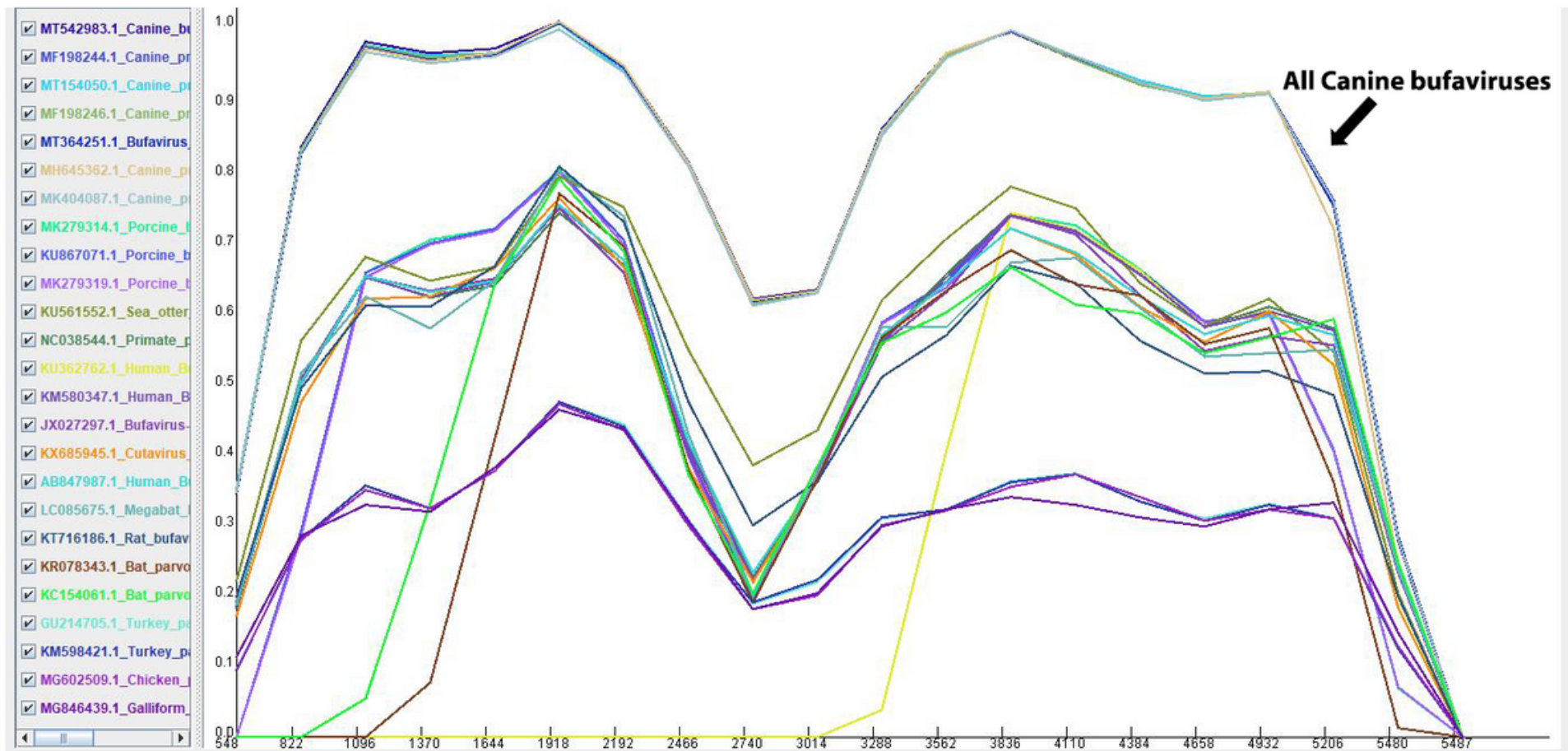

\section{Figure 3}

Recombination patterns of Parvoviridae family based on whole genome sequence from RAT analysis. The cluster of all CaBuVs were indicated with a solid arrow. Recombination events in other BuVs are evident as crossing of lines.

\section{Supplementary Files}

This is a list of supplementary files associated with this preprint. Click to download.

- Supplementarydata.docx

- Table1.xlsx

- Table2a.xlsx

- Table2b.xlsx

- Table3.xlsx 